\title{
Design and application of occupation exposure declaration and monitoring information system for medical staff
}

\author{
Xiaofei Zhang ${ }^{1,2, a}$, Lingli Dai ${ }^{3, b}$, Lu Ren ${ }^{1, c}$, Siyuan Tang ${ }^{1, d, *}$ \\ ${ }^{1}$ Xiangya Nursing School, Central South University, Tongzipo Road,Changsha, China \\ ${ }^{2}$ the Second Hospital of Hebei Medical University, Heping West Road, Shijiazhuang, china \\ ${ }^{3}$ the Third Xiangya Hospital of Central South University, Tongzipo Road,Changsha, China \\ a.hulixifei@163.com, b.616822762@qq.com, c.421579477@qq.com, d.tangsyuan@126.com
}

Keywords: occupational exposure, declaration and monitoring, information system.

Abstract: Objective: To register, classify, evaluate and monitor occupational exposure of the medical staff timely and accurately, improve the efficiency of the occupational exposure declaration and monitoring, so as to minimize the incidence and injury of occupational exposure and guarantee the safety and occupational health of the medical staff, this study attempts to develop and design an occupational exposure declaration and monitoring system software for medical staff with multiple functions and convenient for users, which will be the beneficial supplement of Hospital Information system (HIS).

Methods: Through investigation and documents research, we try to comprehend the procedure of occupational exposure declaration and monitoring, determine the system function demand, and construct a three-layer structure system based on B/S structure model. The whole system is divided into interface layer, function layer and data layer. The overall design technology includes the code of c \# programming language, the software platform of Visual Studio 2008, the development tools of ASP.NET script technology and the database services of SQL Server relational database management system.

Results: This system has 6 function modules, including the declaration module, the query and statistical module, the maintaining module, the evaluation and monitoring module, etc. The declaration module of the system can be used effectively in registration, report of occupational exposure accidents and also provide query and statistical functions. The evaluation and monitoring module can assess the severity level of the exposure automatically according to the further inspection, then provide preventive treatments or measures based on the evaluation results, as well as monitor automatically whether the patient has the symptoms of blood-borne acute infectious diseases. At the same time, this module cane effectively monitors the serological results of the infectious diseases in different periods after exposure, and provides timely and reliable data for further intervention and treatment.

Conclusion: The occupation exposure declaration and monitoring information system for medical staff can realize the input and declaration of basic occupational exposure information, the function of data query, statistics and export with stable and reliable operation. The function of evaluation and monitoring can provide convenient and quick intervention and treatment counter measures after exposure, which provides another important technological platform for minimizing the harm and risk of the occupation exposure. 


\section{Introduction}

Medical staff has the high risk of occupation exposure. When they do their routine work, they are easily infected by microbes, such as hepatitis B, C and HIV, then endanger themselves. Occupation exposure for medical staff is in the period of high incidence. Studies said that the occupation exposure rate in the domestic medical staff is from 62.8 percent to 98.0 percent, whereas that of abroad is from 22.5 percent to 73.0 percent $^{[1,2]}$. One expert surveying 1,075 nurses found that about 80.6 percent nurses had needle-stick injuries, and 54.8 percent needles had been polluted by patients' blood $^{[3]}$. It says that the rate of a person infected by a needle with hepatitis B, hepatitis C and HIV is from 6.0 percent to 30.0 percent, 3.0 percent to 6.0 percent and zero to 0.3 percent, respectively.

Informatization building is a significant technical means to make hospital function fastly and efficiently. At present, hospital information system (HIS) has been an important mark of hospital modernization, and it evolves gradually to a frontier subject involving medicine, information, management, computer and other subjects, and even it is recognized now as an indispensable branch of medical informatics. The HIS system has many subsystem pertain to hospital specific management, besides it can improve work efficiency, reduce the medical staff's workload and serve the patients better with strong technical support , therefore it is now popular and widely used in our country.

The electronic information processing of the occupation exposure registration and declaration procedure is the foundation of occupation exposure declaration and monitor information system ${ }^{[4,5]}$. 2004, China ministry of health issued < the work's guiding principles of HIV and virus occupation exposure for medical staff $>$. The principles request that medical institutions must design and use occupation exposure registration form, report occupation exposure in detail including the basic information about medical staff with occupation exposure, exposure site and position, the type of needles' injury, the grade of exposure, exposure source and intervention after exposure ${ }^{[6]}$.

However, when occupation exposure accident happens, the traditional method is all by medical staff themselves to register, report, query, statistics, intervene and follow. Because the procedure is lengthy and inefficient, the phenomena that medical staff give up reporting or the infected staff do not get treatment always exist. Besides, related managers save, query and analyze related data all by manual which easily made mistake.

So the purpose of the study is to design an information system based on HIS, so that medical staff can report the exposure accident timely, administrative department monitor the exposure easily and make personized preventive measures quickly, finally reduce the exposure incidence .

\section{Demand analysis}

\subsection{Feasibility analysis of the system}

The feasibility of the goal: The study's goal is to develop a medical staff's occupation exposure declaration and monitor information system through hospital intra network based on the existed HIS. The system use B/S three layer structure model, ASP.NET technology, the existed service platform. So, the goal is specific and plausible.

The feasibility of the technology: The overall design technology includes the code of c \# programming language, the software platform of Visual Studio 2008, the development tools of ASP.NET script technology and the database services of SQL Server relational database management system

The feasibility of application: The system development is based on medical conditions and environment, to change and modify the disadvantages of the exiting procedure with informatization, so it will have a good future for practice. 
The controllability of risk: avoid goal ambiguity and technology weakness; focus on information safety.

\subsection{The general demand of the system}

Normative design with standard process, to avoid errors and mistakes; user-friendly process, to save time and improve efficiency;

Timely: Occupation exposure is dangerous to medical staff and their family, so they need to report and convey the related data quickly.

Safety: avoid virus attacked which may lead to system breakdown

Convenience: easy to use; concise interface; reduce their workload

\subsection{Function demand}

Register and login module user register with their ID, login and exist the system.

Declaration module: input the fist-hand occupation exposure data; develop occupation exposure report sheet.

Evaluation module: evaluate exposure source, type of exposure, amount of exposure and severity of exposure, determine the following intervention.

Monitor module: including exposure index database, exposure people database and exposure calculation database.

Result query and print

Change the password

\subsection{Demand for runtime environment and function}

Demand for runtime environment adaptability

CPU: $8.0 \mathrm{GHz}$

Internal storage: $32 \mathrm{~GB}$

Cache: 8GB

Hard disk drive: 500TB

Displayer: VGA color display

Operating system: Windows Server 2008

Software development platform: Visual Studio 2008

Back database: SQL Server

JDK: JDK1.6

Practical demand: friendly interactive interface; function operating easily; good expandability; information sharing.

Function demand: quick response; stable operation; system safety; good fault-tolerance; easy to maintain.

\section{Technical framework analysis}

\section{1. $\mathrm{C} / \mathrm{S}$ and $\mathrm{B} / \mathrm{S}$ structural model}

B/S structure, Browser/Server, is a new network structure model based on Microsoft Web. At present, most system's client design use the browser based on Web model. The typical feature is that it unify the various client, then focuses the core of the system function on the server, so that the 
system's development, maintenance and use are more and more convenient ${ }^{[7]}$. Client just installs a browser, like internet explorer or other widely used browser, whereas Server install various database like SQL Server, the function of Web Server is to realize the data exchange or asynchronous transmission between the browser and the database. B/S structure basic operation principle is that the Client operates according to orders, and transit the requirement to the Server through web, then the results returned form the Server show on the interface of the Client I in a certain way.

ASP.NET is a script technology embedded into the web page. Active Server Pages (ASP) was first developed by Microsoft company, with the feature of simpleness and customization, and now rise rapidly in the trend of website development. At present, it has many editions since the first edition in 2002.

3.2 SQL Server database technology Structured Query Language is a relational database management system, was developed by Microsoft, Sybase and Ashton-Tate companies. The main function is to build connection between different database and bridge between the interface layer and database. SQL command have many operations, like updating the database and extracting data from the database. And now the concept of SQL has been refined and the function has been expanded, and is popular now.

\section{Design and application}

\subsection{The design of overall function structure and procedure}

Based on the demand analysis result about occupation exposure declaration and monitor system, $\mathrm{B} / \mathrm{S}$ three plyer architecture design was used to design the system. In the architecture, the main work interface worked through browser, the main processing work focused on the server side (server), and only a small amount of logical transaction work assigned to the front (browser). The design would simplify the client's computer load greatly, and reduce the cost and workload of system maintenance and upgrade under Internet/Intranet model. When using the architecture, the system should provide functions such as software login, new adding report, data query and statistics, evaluate and monitor, item and parameters, permission setting and password modification, etc. The following is the system's function structure:

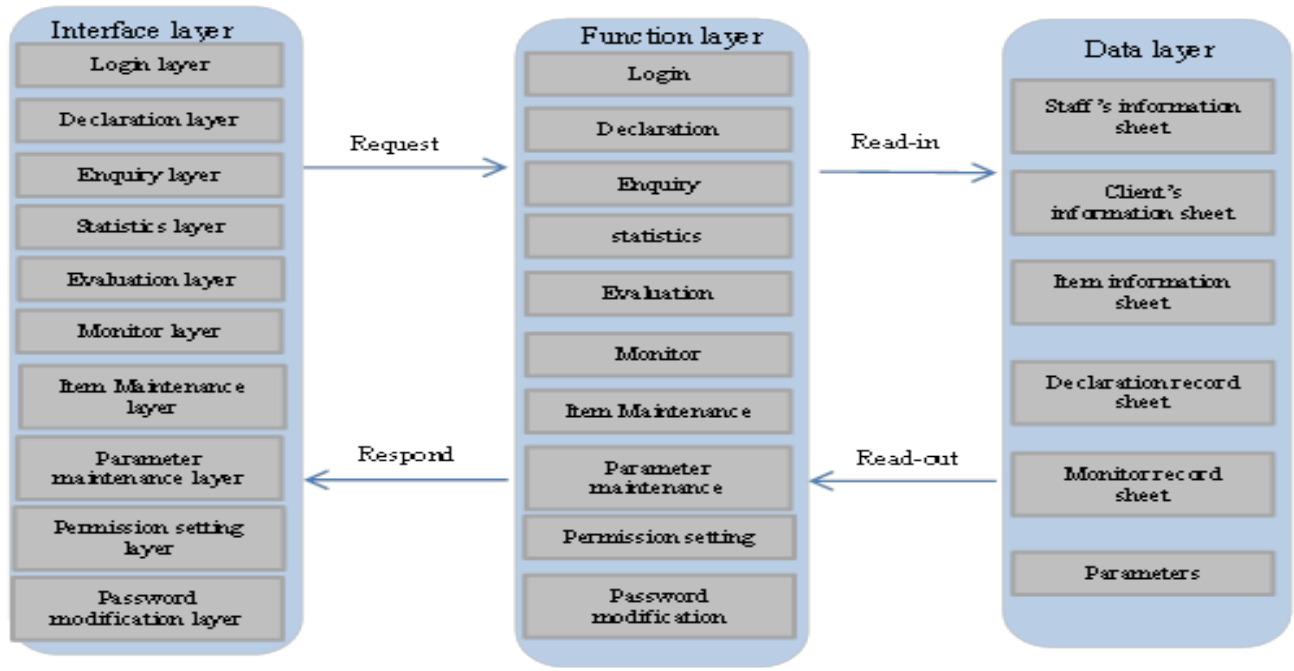

Fig1. The function structure of occupation exposure declaration and monitor information system for medical staff. 


\subsection{The design of function module}

According to the analysis of the demand and the whole system procedure, the system can be divided into six different and interrelated modules, including the module of login, new report, enquiry and statistics, item maintenance, evaluation and monitor and password modification. The following graph shows their correlation.

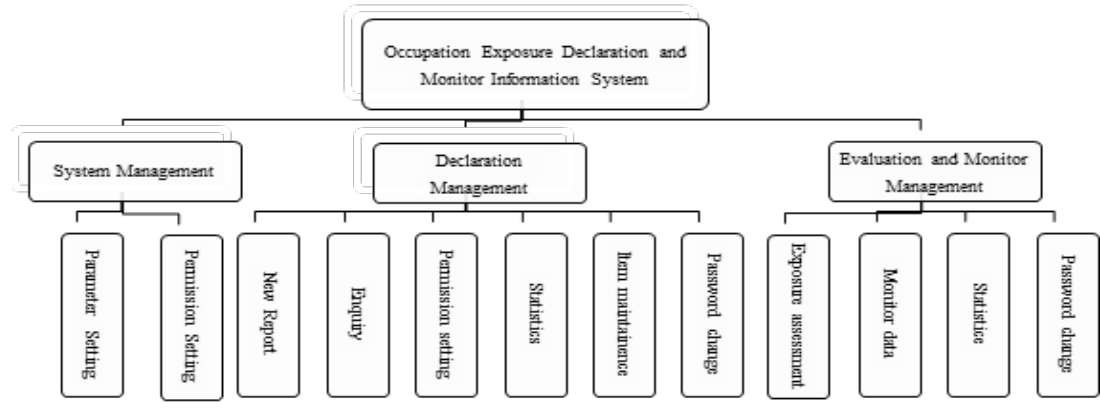

Fig 2. The function module structure of occupation exposure declaration and monitor information system for medical staff

\subsection{The application of each function module and database of the system}

We designed the procedure and realization means of each function module in detail. During design, specific procedure and computer code were used to realize the target goal and present a good and user-friendly interface and usage.

Database is the core and basal part of the whole system. The large amount of data needed by the system should be organized in a certain model so that it can be called and gained systematically according to de needs, then provide the function of storage, maintenance, enquiry, and etc. The main database was the basic information database form of exposure report, including the ordinary information about the exposure history; another was he basic information database form of exposure evaluation and monitor, involving information about exposure details requested by the principles.

\section{Conclusions}

According to the essential process of software development, the study did the following work: fist, we analyzed the system feasibility by user function and non-function needs and runtime environment, so define the boundary; then, we designed system technology architecture based on web three layer frame under B/S model after comparing B/S AND C/S and analyzing the technology of popular front end and service-side; finally, we designed the exposure report and monitor system, database, and make the whole system realized.

Future study will: collect occupation exposure information automatically; develop related app system; do the big data mining.

\section{Reference}

[1] Schmid K, Schwager C, Drexler H, Needle stick injuries and other occupational exposures to body fluids among fist employees and medical students of a German University: Incidence and follow-up [J]. Journal of Hospital Infection, 2007, 65:124-130.

[2] Hong Zhou, Wei Zheng , Fangzheng Han ,et al. Study on Occupational Exposure and Preventive Strategies in 
Medical Staff by Prospective Surveillance [J]. Chinese Journal of Nosocomiology,2010,20(12):1715-1716.

[3]Xiaodong Gao, Bijie Hu, Wenjuan Wang, et al. Sharp injuries among healthcare workers in 70 shanghai hospitals [J]. Chinese Journal of Nosocomiology, 2010, 20(12):1713-1714.

[4]Jamia. Development of a Clinical Data Warehouse for Hospital Infection Control[J]. Journal of the American Medical Informatics Association,2003,10 (5):454-462.

[5]Lijie liu. Design of a large hospital nosocomial infection monitoring information system[J]. The Chinese journal of clinical medical research, 2007,13(5):698.

[6] Chunlang Dai. Design and implementation of occupational exposure declaration system for medical staff based on .NET technology [J] . China Medical Devices, 2014, 29(03): 51-54.

[7]Haifan Zhang. Introduction to software engineering [M]. Fourth edition, Beijing: Tsinghua University Press, 2005. 